\title{
THE WEAK HAUPTVERMUTUNG FOR CELLS AND SPHERES
}

\author{
BY HERMAN GLUCK
}

Communicated by A. W. Tucker, March 28, 1960

THEOREM. If $P$ and $Q$ are two triangulations of the $n$-sphere (closed $n$-cell), there is a third triangulation $M$ which can be obtained from either by subdivision. In fact, $M$ can be obtained from either $P$ or $Q$ by subdivision of a single $n$-simplex.

The following result, obtained recently by M. Brown [1], is the principal tool of both proofs.

LEMMA. Let $S^{n-1}$ be an $n-1$ sphere embedded in the $n$-sphere $S^{n}$. If $S^{n-1}$ has a neighborhood in $S^{n}$ homeomorphic to $S^{n-1} \times[-1,1]$, in which $S^{n-1}$ is embedded as $S^{n-1} \times 0$, then the closures of the complementary domains of $S^{n-1}$ in $S^{n}$ are both closed $n$-cells.

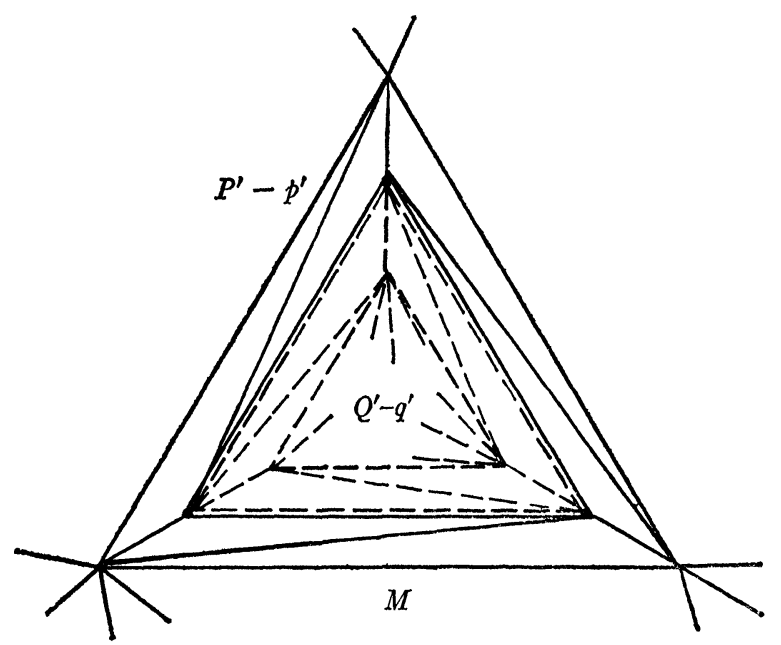

FIG. 1

We prove the theorem first for the $n$-sphere. Let $p$ be an $n$-simplex of $P, q$ an $n$-simplex of $Q$. Let $p^{\prime}$ be a smaller, concentric $n$-simplex inside $p$, and let $P^{\prime}$ be obtained from $P$ by drawing $p^{\prime}$ inside $p$ and triangulating the region $\left(S^{n-1} \times[0,1]\right)$ between the boundaries of $p$ and $p^{\prime}$. Similarly for $q^{\prime}$ and $Q^{\prime}$. The boundaries of $\left|p^{\prime}\right|$ and $\left|q^{\prime}\right|$ have neighborhoods as required in the lemma, so they split $\left|P^{\prime}\right|$, resp. $\left|Q^{\prime}\right|$, into two closed $n$-cells, one of which is $\left|p^{\prime}\right|$, resp. $\left|q^{\prime}\right|$, and the 
other $\left|P^{\prime}-p^{\prime}\right|$, resp. $\left|Q^{\prime}-q^{\prime}\right|$. Let $\omega$ be a simplicial homeomorphism sending the boundary of $p^{\prime}$ onto the boundary of $q^{\prime}$. The complex $M=\left(P^{\prime}-p^{\prime}\right) \bigcup_{\omega}\left(Q^{\prime}-q^{\prime}\right)$, which clearly triangulates an $n$-sphere, can be obtained from $P$ by subdivision of $p$, and from $Q$ by subdivision of $q$. Figure 1 illustrates the construction of $M$.

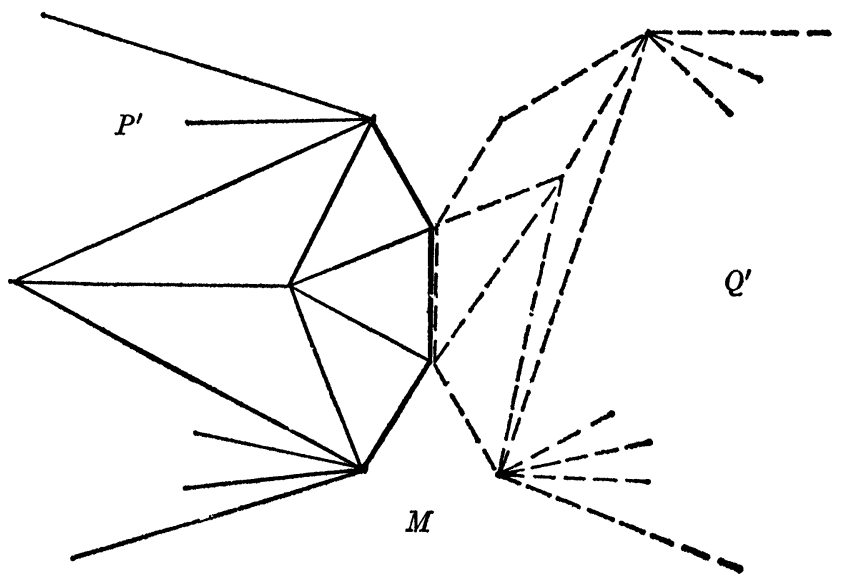

FIG. 2

The closed $n$-cell is treated similarly. Let $p$ be an $n-1$ simplex on the boundary of $|P|$, and let $\alpha$ be the vertex opposite $p$ in the $n$ simplex $\alpha \circ p$ of $P$ containing $p$. Let $p^{\prime}$ be drawn inside $p$ and concentric with it, and triangulate the region $\left(S^{n-2} \times[0,1]\right)$ between the boundaries of $p$ and $p^{\prime}$. Let $\alpha^{\prime}$ be the barycenter of $\alpha \circ p$. Joining $\alpha^{\prime}$ to the already subdivided boundary of $\alpha \circ p$ yields a subdivision $P^{\prime}$ of $P$. The $n$-simplex of $P^{\prime}$ containing $p^{\prime}$ as a face is $\alpha^{\prime} \circ p^{\prime}$. The boundary of $\left|p^{\prime}\right|$ has a neighborhood on the boundary of $\left|P^{\prime}\right|$ which satisfies the conditions of the lemma. It thus splits the boundary of the $n$-cell into two closed $n-1$ cells with a common boundary. Let the same steps be taken with the complex $Q$, leading to subdivision $Q^{\prime}$ containing an $n-1$ simplex $q^{\prime}$ on the boundary of $\left|Q^{\prime}\right| . q^{\prime}$ will, in turn, be a face of the $n$ simplex $\beta^{\prime} \circ q^{\prime}$ of $Q^{\prime}$.

Let $\omega$ be a simplicial homeomorphism sending $p^{\prime}$ onto $q^{\prime}$. Setting $M=P^{\prime} \bigcup_{\omega} Q^{\prime}$, we notice that an immediate consequence of the fact that the boundary of $\left|p^{\prime}\right|$, resp. $\left|q^{\prime}\right|$, splits the boundary of $\left|P^{\prime}\right|$, resp. $\left|Q^{\prime}\right|$, into two closed $n-1$ cells is that $|M|$ is a closed $n$-cell. Furthermore, $\alpha^{\prime} \circ p^{\prime} \bigcup_{\omega} Q^{\prime}$ is isomorphic to a subdivision of $\alpha^{\prime} \circ p^{\prime}$, and $P^{\prime} \bigcup_{\omega} \beta^{\prime} \circ q^{\prime}$ to a subdivision of $\beta^{\prime} \circ q^{\prime}$ by a similar argument. $M$ can therefore be obtained from $P$ by subdivision of $\alpha \circ p$ and from 
$Q$ by subdivision of $\beta \circ q$. Figure 2 exhibits the construction of $M$.

Our method does not demonstrate the combinatorial equivalence of $P$ and $Q$, and is therefore only a verification of a weakened form of the full Hauptvermutung (see [2]). We observe, however, that the subdivision of $P$ into $M$ is as "nice" as is the complex $Q$, and the subdivision of $Q$ into $M$ is as "nice" as is the complex $P$. If, for example, $Q$ is a combinatorial cell or sphere, then $P$ is combinatorially equivalent to $M$. If both $P$ and $Q$ are combinatorial cells or spheres, we obtain a special case of a classical result due to Newman, [3].

\section{REFERENCES}

1. M. Brown, $A$ proof of the generalized Schönflies theorem, Notices Amer. Math. Soc. vol. 7 (1959) p. 783.

2. R. Furch, Zur Grundlegung der kombinatorischen Topologie, Abh. Math. Sem. Hansischen Univ. vol. 3 (1924) pp. 69-88.

3. M. H. A. Newman, On the superposition of n-dimensional manifolds, J. London Math. Soc. vol. 2 (1927) pp. 56-64.

Princeton University 